\title{
Azelnidipine suppresses the progression of aortic aneurysm in wild mice model through anti-inflammatory effects
}

\author{
Hirotsugu Kurobe, MD, PhD, ${ }^{\text {a,d }}$ Yuki Matsuoka, BS, ${ }^{a}$ Yoichiro Hirata, MD, PhD, ${ }^{\text {b,c }}$ Noriko Sugasawa, BS, ${ }^{a}$ \\ Mark W. Maxfield, MD, ${ }^{\mathrm{d}}$ Masataka Sata, MD, $\mathrm{PhD},{ }^{\mathrm{c}}$ and Tetsuya Kitagawa, MD, $\mathrm{PhD}^{\mathrm{a}}$
}

\begin{abstract}
Background: Although systemic hypertension is closely associated with aortic aneurysm (AA) formation, there are many patients with AA without hypertension. In these patients, an inflammation-mediated progression of aneurysmal disease is likely responsible for AA growth and eventual rupture. Unfortunately, there remains no reproducible and durable small animal model of aortic aneurysmal disease, the development of which would enable the investigation of the pathophysiology of this vexing condition. The first aim was to establish a useful wild-type mouse model of AA with low mortality. The second aim was to use this model to assess the protective effect of azelnidipine, a new calcium channel blocker, against the progression of the AA independent of its antihypertensive effect.
\end{abstract}

Methods: Angiotensin II and $\beta$-aminopropionitrile (a lysyl oxidase inhibitor) were administrated subcutaneously in 7-week-old C57BL/6J mice using an osmotic minipump for 4 weeks to generate a wild-type mouse model of AA. Concurrently, azelnidipine (a calcium channel blocker) or a placebo was administrated orally for 4 weeks. Mice were humanely killed and assessed at the end of the 4 weeks of pharmacologic manipulation.

Results: The combined infusion of angiotensin II and $\beta$-aminopropionitrile induced degenerative aneurysm of the thoracic and/or abdominal aorta $(11 / 12 ; 92 \%)$. The majority of aneurysms were located in the distal aortic arch and suprarenal abdominal aorta. Although there was no difference in systolic blood pressure between the control and azelnidipine-treated groups, azelnidipine significantly reduced the incidence of AA $(2 / 11 ; 18 \%)$. Azelnidipine treatment reduced the pathologic findings normally associated with aneurysm formation within the aortic wall. Azelnidipine also reduced the number of macrophage antigen-3 (MAC-3)-positive cells in the periaortic adipose tissue and reduced the gene expression levels of tumor necrosis factor-alpha and matrix metalloproteinase-2 and -9 within the aortic wall.

Conclusions: This study demonstrates that combined treatment with angiotensin II and $\beta$-aminopropionitrile induces degenerative AAs in wild-type mice, and azelnidipine prevents aneurysm progression via its anti-inflammatory effect. (J Thorac Cardiovasc Surg 2013;146:1501-8)

The incidence of aortic aneurysmal disease is increasing, owing to an aged population and improved screening methods. ${ }^{1-3}$ As less invasive methods of endovascular treatment of aortic aneurysms (AAs) become more common and outcomes improve, open surgery is no longer the only option for patients, which allows treatment of aneurysmal disease in high-risk patients and in those with hostile abdomens. ${ }^{4-6}$

\footnotetext{
From the Departments of Cardiovascular Surgery ${ }^{\mathrm{a}}$ and Cardiovascular Medicine, ${ }^{\mathrm{b}}$ Institute of Health Biosciences, University of Tokushima Graduate School, Tokushima, Japan; the Department of Pediatrics, ${ }^{\mathrm{c}}$ Graduate School of Medicine, University of Tokyo, Tokyo, Japan; and the Department of Surgery, ${ }^{\text {d }}$ Yale University School of Medicine, New Haven, Conn.

This study was funded by a Grant-in-Aid for Scientific Research from Japan Society for the Promotion of Science.

Disclosures: Authors have nothing to disclose with regard to commercial support. H.K. and Y.M. contributed equally to this work.

Received for publication Jan 16, 2013; revisions received Feb 18, 2013; accepted for publication Feb 28, 2013; available ahead of print March 26, 2013.

Address for reprints: Hirotsugu Kurobe, MD, PhD, Institute of Health Biosciences, The University of Tokushima Graduate School, Department of Cardiovascular Surgery, 3-18-15 Kuramoto-chou, Tokushima-shi, Tokushima 770-8503, Japan (E-mail: hirotsugu.kurobe@clin.med.tokushima-u.ac.jp). $0022-5223 / \$ 36.00$

Copyright (c) 2013 by The American Association for Thoracic Surgery http://dx.doi.org/10.1016/j.jtcvs.2013.02.073
}

Unfortunately, there are no pharmacologic agents that retard the growth of AAs. After identification of an aortic aneurysmal lesion, physicians treat the patient with antihypertensive medications and conduct serial ultrasonographic examinations until the aneurysm grows to a size at which intervention is indicated. Although it has been demonstrated both clinically and experimentally that systemic hypertension is closely associated with AA formation, there are certainly many patients with AAs who are normotensive. ${ }^{7-9}$ Therefore, the establishment of an experimental model of AAs that is independent of hypertension may provide insights into aneurysm formation and propagation, which most importantly may lead to novel targets of therapeutic intervention. ${ }^{10}$

The degeneration or disorganization of the elastic lamina within the aortic wall that occurs with aging is closely associated with AA progression..$^{7-9,11,12}$ The extracellular matrix of the aortic wall is predominantly composed of collagen and elastin fibers, which are enzymatically cross-linked by lysyl oxidase. ${ }^{13}$ Previous studies have demonstrated that the activity of lysyl oxidase decreases with aging ${ }^{14,15}$ and that degeneration of elastic fibers is induced by 


\section{Abbreviations and Acronyms \\ $\mathrm{AA}=$ aortic aneurysm \\ BAPN $=\beta$-aminopropionitrile \\ EVG = elastica van Gieson \\ $\mathrm{H} \& \mathrm{E}=$ hematoxylin and eosin \\ MMP = matrix metalloproteinase \\ RT-PCR $=$ reverse-transcriptase polymerase chain reaction \\ TNF- $\alpha=$ tumor necrosis factor-alpha}

$\beta$-aminopropionitrile (BAPN), an inhibitor of lysyl oxidase. ${ }^{16-18}$ Recently, a mouse model of thoracic and abdominal AAs has been developed using the combination of angiotensin II-induced hypertension and BAPN-induced elastic lamina degeneration; however, the study was limited by a prohibitively high mortality rate. ${ }^{16}$

Therefore, the first aim of this study is to establish a useful wild-type mouse model of AA with low mortality. The second aim of this study is to assess the protective effect of azelnidipine against the progression of AA independent of its antihypertensive effect. Azelnidipine is a newly developed $\mathrm{Ca}^{2+}$ channel blocker and has been demonstrated to have an anti-inflammatory effect independent of its antihypertensive effect. ${ }^{19-22}$

\section{METHODS}

All animal experiments were conducted in accordance with the "Guide for the Care and Use of Laboratory Animals" (National Institutes of Health Publication No. 85-23, revised 1996). The Institutional Review Board at Tokushima University approved all animal procedures.

\section{Induction of AA and Azelnidipine Treatment}

Seven-week-old C57BL/6J male mice (CLEA Japan, Inc, Tokyo, Japan) were used. A placebo vehicle or a constant infusion of angiotensin II (1000 $\mathrm{ng} / \mathrm{kg}$ body weight per minute $)$ and BAPN $(37.5 \mathrm{mg} / \mathrm{kg} / \mathrm{d})$ were administrated through a subcutaneously implanted osmotic pump (Alzet Osmotic Pumps; Durect Corp, Cupertino, Calif) for 4 weeks. ${ }^{23,24}$ In our study, after preliminary testing 3 different doses of BAPN $(37.5,75$, and $150 \mathrm{mg} / \mathrm{kg} / \mathrm{d})$, it was determined that $37.5 \mathrm{mg} / \mathrm{kg}$ led to rates of AA development of $90 \%$ to $100 \%$ with a survival of $80 \%$. Therefore, we modified our protocol and used BAPN at a dose of $37.5 \mathrm{mg} / \mathrm{kg}$ and the same dose of $1000 \mathrm{ng} / \mathrm{kg} / \mathrm{min}$ angiotensin II that was used previously by Kanematsu and associates. ${ }^{16}$ Mice were also administered concurrently either oral azelnidipine $\left(0.5 \mathrm{mg} / \mathrm{kg} / \mathrm{d}^{25}\right.$; Daiichi Sankyo Co, Ltd, Tokyo, Japan) or a placebo for 4 weeks by gavage once a day (azelnidipine vs control).

\section{Measurement of Blood Pressure}

Systolic blood pressure was measured weekly using a tail-cuff method (BP-98A, Softron, Japan) during the 4-week experimental period when mice were undergoing pharmacologic administration and is presented here as the average of 10 measurements. ${ }^{23}$

\section{Tissue Harvesting and Macroanalyzing}

Animals were housed in a pathogen-free facility under climate-controlled conditions with a 12-hour light/12-hour dark cycle and were provided with standard diet chow (Oriental Yeast Co, Tokyo Japan) and water ad libitum.
At the end of the 4-week drug infusion period, these mice were perfused with cold phosphate-buffered saline $(20 \mathrm{~mL})$ under physiologic pressure, and then the aorta between the heart and the aortic bifurcation and periaortic fat tissue were harvested and used for tissue analysis and quantitative reverse-transcriptase polymerase chain reaction (RT-PCR) analysis.

AAs were defined morphologically as a localized dilation of the aorta with a maximal outer diameter larger than $50 \%$ of its adjacent intact portion of the aorta, which are the same criteria used for defining human AAs. ${ }^{16}$ Images were analyzed using cellSens software (version 1.41; Olympus Corporation, Tokyo, Japan) to measure the outer diameter of the aorta.

\section{Histologic and Immunohistochemical Examinations}

The harvested tissues were fixed in $4 \%$ paraformaldehyde and then embedded in paraffin. Serial cross sections $(5-\mu \mathrm{m}$ thick) from both the thoracic and abdominal aortas were mounted on microscope slides and examined histologically and immunohistochemically. The sections were stained with hematoxylin and eosin (H\&E) and elastica van Gieson (EVG).

Mac-3 (purified rat anti-mouse Mac-3 antibody; 550292; BD Pharmingen, BD Biosciences, San Jose, Calif) immunohistochemical staining was performed to identify macrophages in the aortic wall and perivascular adipose tissue. Antibody distribution was visualized using the avidin-biotin complex technique and Vector Red substrate (Vector Laboratories, Inc, Burlingame, Calif).

\section{Quantitative RT-PCR by SYBR Green Detection Method}

The harvested tissues were stored immediately using RNAlater (Life Technologies Corporation, Carlsbad, Calif). Total RNA was isolated from the aortic tissue using TRIzol (Life Technologies Corporation) in accordance with the manufacturer's instructions. Tumor necrosis factor-alpha (TNF- $\alpha$; an inflammatory cytokine), matrix metalloproteinases (MMP-2 and MMP-9; regulating factors involved in degeneration of the aortic wall), and SIRT-1 (an anti-aging molecule) ${ }^{26}$ mRNA expression levels were determined by RT-PCR analysis (StepOne Real-Time PCR System; Life Technologies Corporation). $\beta$-Actin was used as the housekeeping gene. SYBR green was used to detect $\beta$-actin. Results were expressed as arbitrary units in comparison with the mRNA expression level of $\beta$-actin.

\section{Primer Sequences}

The primer sequences were as follows: mouse TNF- $\alpha$ (sense $5^{\prime}$-CCA GACCCTCACACTCAGATC- $3^{\prime}$ and antisense $5^{\prime}$-CACTTGGTGGTTTG CTACGAC- $3^{\prime}$ ), mouse MMP-2 (sense $5^{\prime}$-GATAACCTGGATGCCGTCG TG- $3^{\prime}$ and antisense $5^{\prime}$-CTTCACGCTCTTGAGACTTTGGTTC- $3^{\prime}$ ), mouse MMP-9 (sense 5'-GCCCTGGAACTCACACGACA- $3^{\prime}$ and antisense $5^{\prime}$-TTGGAAACTCACACGCCAGAAG- $3^{\prime}$ ), and mouse SIRT-1 (sense $5^{\prime}$-TGCAGACGTGGTAATGTCCAAAC- ${ }^{\prime}$ and antisense $5^{\prime}$-ACATCTT GGCAGTATTTGTGGTGAA - $3^{\prime}$ ).

As a housekeeping gene, $\beta$-actin was amplified with the following primer set: sense $5^{\prime}$-CATCCGTAAAGACCTCTATGCCAAC- $3^{\prime}$ and antisense 5'-ATGGAGCCACCGATCCACA - $3^{\prime}$.

\section{Statistical Analyses}

Data are presented as mean \pm standard error. To analyze variations between groups, we used the nonparametric Mann-Whitney test for paired data. One-way analysis of variance with the post hoc (Newman-Keuls) test was used to compare variations within groups.

\section{RESULTS}

Combined Infusion of Angiotensin II and BAPN Induced Hypertension and AA Formation

The systolic blood pressure was higher after infusion than before infusion. Administration of azelnidipine had no 

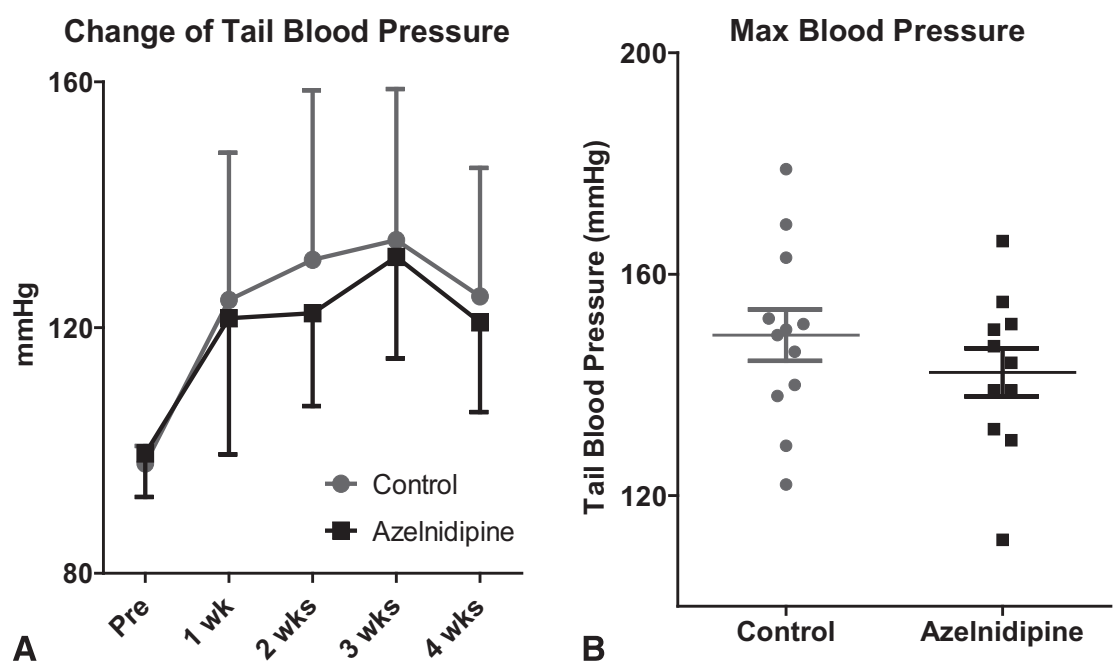

FIGURE 1. Blood pressure measurements. A, Changes over time in systolic blood pressure during the infusion of angiotensin II and BAPN. There is no significant difference between groups ( $\mathrm{n}=12$ in control group, $\mathrm{n}=11$ in azelnidipine group). B, Maximum blood pressure of each mouse after 4 weeks of combination infusion therapy ( $\mathrm{n}=12$ in control group; $\mathrm{n}=11$ in azelnidipine group). $B A P N, \beta$-Aminopropionitrile.

statistically significant effect on either mean systolic or maximum systolic blood pressure between the 2 groups (Figure $1, A$ and $B$ ).

Among the mice that received the combined infusion of angiotensin II and BAPN and were not treated with azelnidipine, 12 of 13 survived until the end of the pump infusion, that is, 4 weeks, with 1 mouse dying prematurely owing to dissecting AA. Of the surviving mice in this group, $91 \%$ (11/12) had AA develop (Figure 2, $A$ and $B$ ). Regarding the type of AA that developed, $50 \%(6 / 12)$ had thoracic AA (Figure 2, $C$ ) and $67 \%(8 / 12)$ had abdominal AA (Figure $2, D)$. Three of the $12(25 \%)$ surviving mice had both thoracic and abdominal AAs.

\section{Azelnidipine Reduced the Incidence of AA Formation}

Azelnidipine treatment in mice receiving the combined infusion of angiotensin II and BAPN significantly reduced the incidence of AA in all aortic regions compared with vehicle treatment (azelnidipine vs vehicle; $18 \%$ vs $91 \%$; $P=.004$ ) (Figure 2, B). The incidences of thoracic and abdominal AAs was decreased by azelnidipine treatment from $50 \%(6 / 12)$ to $0 \%(0 / 11)(P=.02)$ and from $67 \%(8 / 12)$ to $18 \%(2 / 11)(P=.04)$, respectively (Figure 2, $C$ and $D)$.

\section{Azelnidipine Reduced Degenerative Lesions in the Aortic Wall}

Histologic findings of $\mathrm{H} \& \mathrm{E}$ staining demonstrated that combination infusion of angiotensin II and BAPN resulted in mononuclear cell infiltration of the adventitia and media and also in adventitial thickening of the aortic wall compared with normal mice (Figure 3, A). Treatment of mice with azelnidipine concurrently with combination infusion attenuated these findings.
EVG staining showed that combination infusion of angiotensin II and BAPN accelerated degeneration and destruction of elastic layers and adventitial thickening of the aortic wall (Figure 3, B). Azelnidipine rescued the mouse aortas by preventing degenerative lesions within the elastic layer, resulting in the aortic wall resembling that of normal tissue (Figure 3, $B$ ).

\section{Macrophage Infiltration was Inhibited by Azelnidipine Administration}

Mac-3 immunohistochemical staining was performed to analyze macrophage distribution within the aortic wall and in periaortic fat (Figure 4, A). In the aortic wall of mice with the combination-infusion-induced AAs, numerous macrophages were observed in the adventitia and media. The number of macrophages in the arterial wall and perivascular fat tissue was significantly decreased in mice treated concurrently with azelnidipine (Figure 4, B).

\section{Azelnidipine Inhibited TNF- $\alpha$ and MMP-2, -9 Gene Expression in Aortic Wall}

The gene expression levels of TNF- $\alpha$, an inflammatory cytokine, and MMP-2 and MMP-9 in the arterial wall tissue were evaluated by RT-PCR analysis. These gene expression levels were increased by the combined infusion of angiotensin II and BAPN. Azelnidipine treatment attenuated this finding and was found to be of statistical significance for TNF- $\alpha$, MMP-2, and MMP-9 (Figure 5, $A, B$, and $C$ ).

\section{Azelnidipine Increased Vascular SIRT-1 Gene Expression Level in AA Wall}

The vascular SIRT-1 (an antiaging molecule) gene expression level in the aortic wall with aneurysm was not 

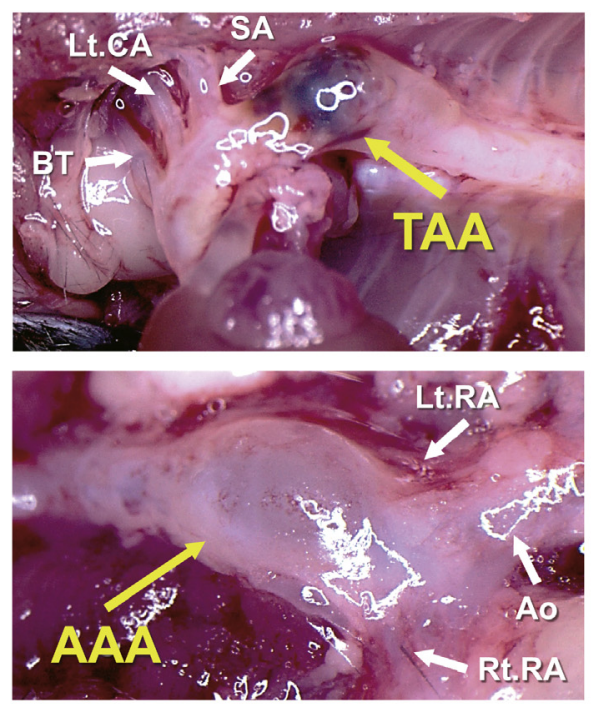

A

Aorta Ratio

(Thoracic aortic lesion)

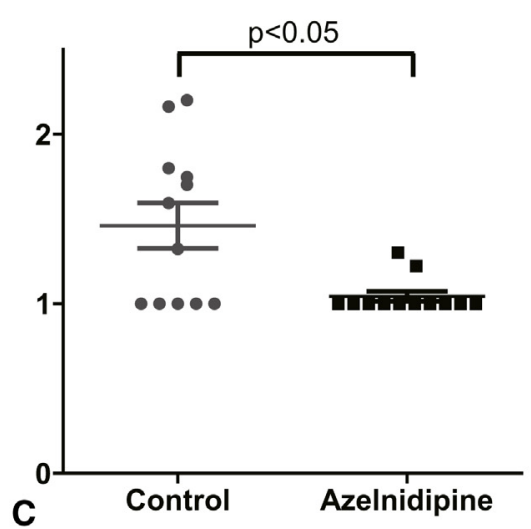

Aorta Ratio

(all aortic lesion)

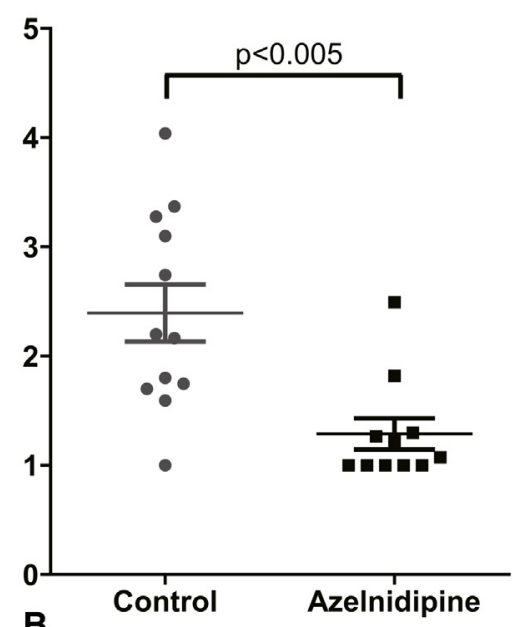

B

Aorta Ratio

(abdominal aortic lesion)

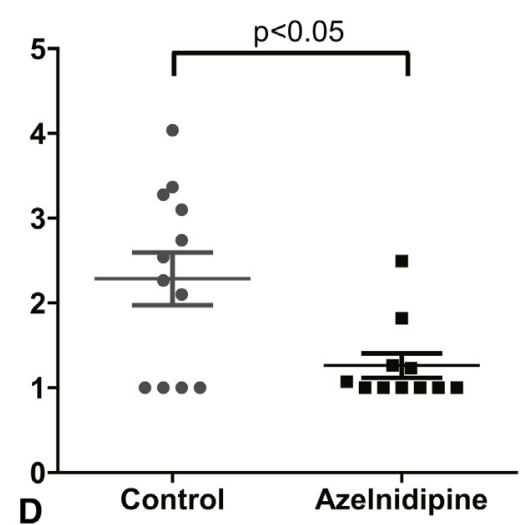

FIGURE 2. Morphologic changes of aorta after combination infusion with angiotensin II and BAPN. A, Representative images of aortic aneurysm. Thoracic aortic aneurysm in upper panel. Abdominal aortic aneurysm in lower panel. BAPN, $\beta$-Aminopropionitrile; TAA, thoracic aortic aneurysm; $A A A$, abdominal aortic aneurysm; $B T$, brachiocephalic trunk; $C A$, carotid artery; $S A$, subclavian artery; $R A$, renal artery; $A o$, aorta; $L t$, left; $R t$, right. B to D, Aortic ratio in each mouse. B, All aortic lesions. C, Thoracic aortic lesions. D, Abdominal aortic lesions. ( $\mathrm{n}=12$ in control group, $\mathrm{n}=11$ in azelnidipine group.)

increased by combination infusion with angiotensin II and BAPN. However, azelnidipine treatment significantly increased the gene expression level of SIRT-1 in the AA wall (Figure 5, D).

\section{DISCUSSION}

Aortic aneurysmal disease is an atherosclerotic disease that affects elderly persons and is more common in men than in women. ${ }^{27,28}$ Despite the increased rate of diagnosis of this disease in recent years, ${ }^{1}$ many patients do not fulfill the criteria for surgical treatment and are therefore managed by medical treatment and serial computed tomographic and ultrasound examinations. Unfortunately, few medications have been demonstrated to inhibit the progression of aortic aneurysmal disease besides antihypertensives. A significant obstacle to drug development for this disease is the lack of a reliable and reproducible animal model. The aims of this study, therefore, are twofold: to establish a small animal model of aortic aneurysmal disease without genetic modification and to evaluate a novel pharmacologic agent for the treatment of this entity.

Kanematsu and associates ${ }^{16}$ demonstrated that combination of angiotensin II-induced hypertension and lysyl oxidase inhibition with BAPN induces AA formation in C57BL/6J mice. However, this study was limited by a prohibitively high mortality rate during the experimental period, which may have been related to high levels of BAPN that led to rapid degeneration of the aortic wall. Therefore, we modified our protocol after preliminary BAPN-dose testing and used BAPN at a dose of $37.5 \mathrm{mg} / \mathrm{kg}$ and the same dose of $1000 \mathrm{ng} / \mathrm{kg} / \mathrm{min}$ 

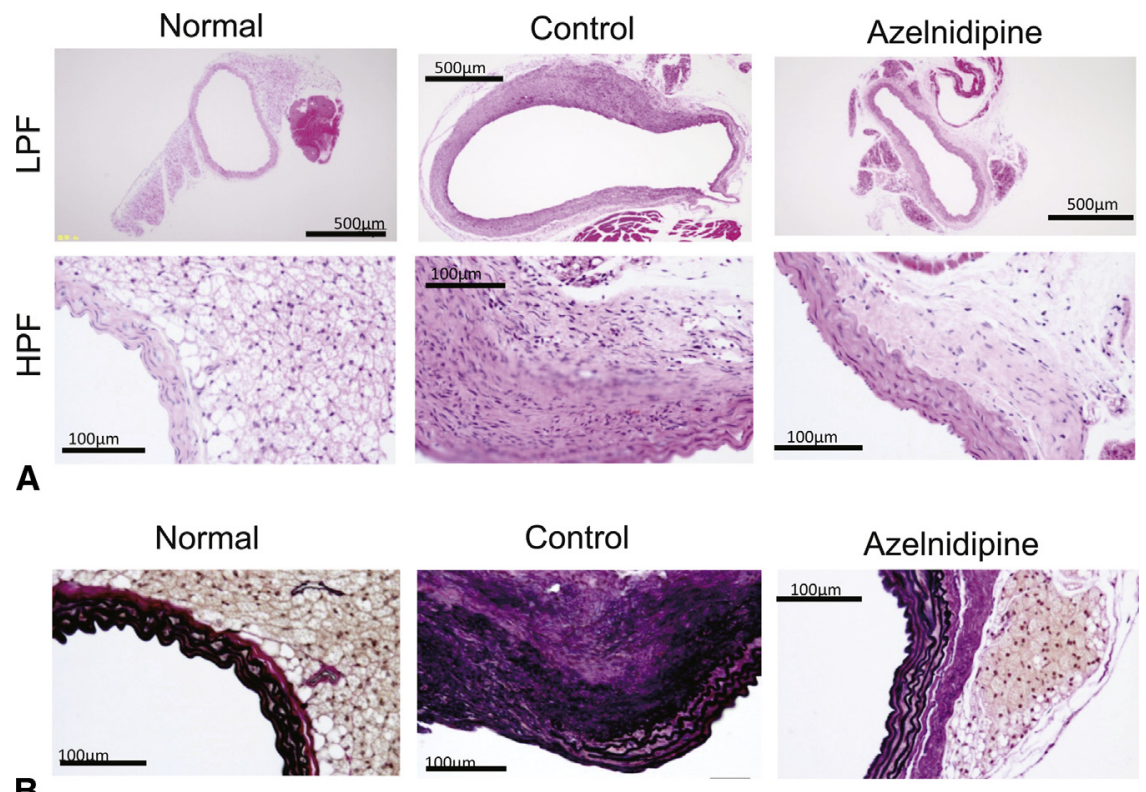

FIGURE 3. Histologic analysis of aorta. A, Hematoxylin and eosin (H\&E) staining of aorta. $L P F$, Low-power field (bar shows length in $500 \mu \mathrm{m})$; $H P F$, high-power field (bar shows $100 \mu \mathrm{m}$ of length). B, Elastica van Gieson (EVG) staining of aorta (bar shows $100 \mu \mathrm{m})$.

angiotensin II that was used previously by Kanematsu and colleagues, ${ }^{16}$ and we administered these drugs by infusion via a subcutaneous osmotic pump. Thus we obtained rates of AA development of $90 \%$ to $100 \%$ with a survival of $80 \%$. Our experimental period was 4 weeks, which was shorter than theirs, and our study after
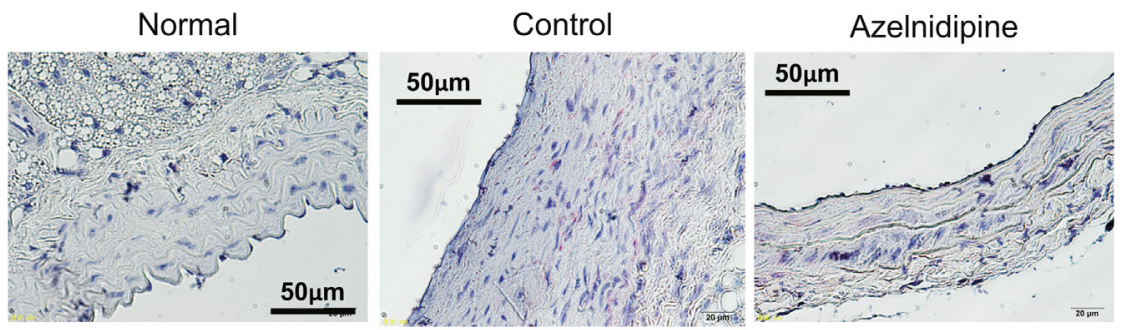

A

MAC3 Positive Cells
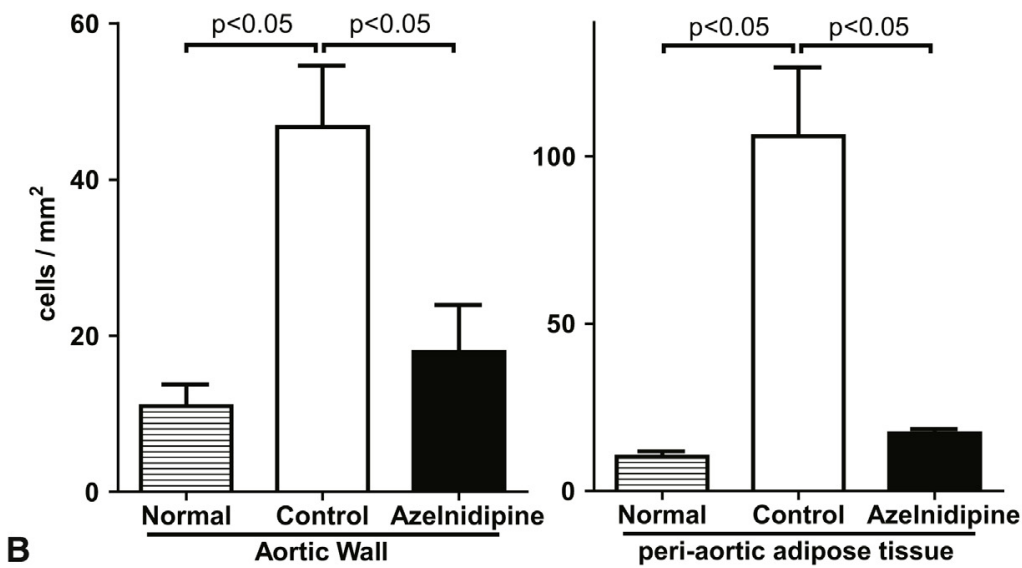

FIGURE 4. Mac-3 immunohistochemical staining of aorta. A, Representative images of Mac-3 immunohistochemical staining (bar shows $50 \mu \mathrm{m}$ of length). B, The number of Mac-3-positive cells in aortic lesions was counted in the control group (treated with a combination infusion of angiotensin II and BAPN), azelnidipine group (treated with the combination infusion plus azelnidipine administration), and normal group (nontreated). Left panel: aortic wall; right panel: periaortic adipose tissue; $\mathrm{n}=7$ in each group. $B A P N, \beta$-Aminopropionitrile. 
TNF- $\alpha$

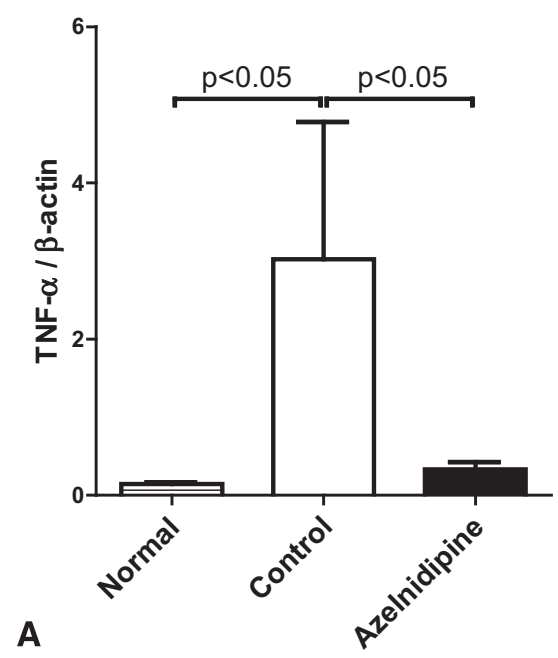

MMP-9

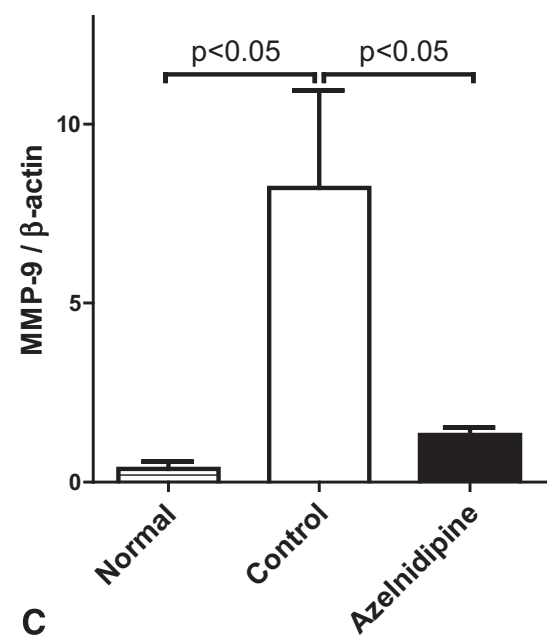

MMP-2

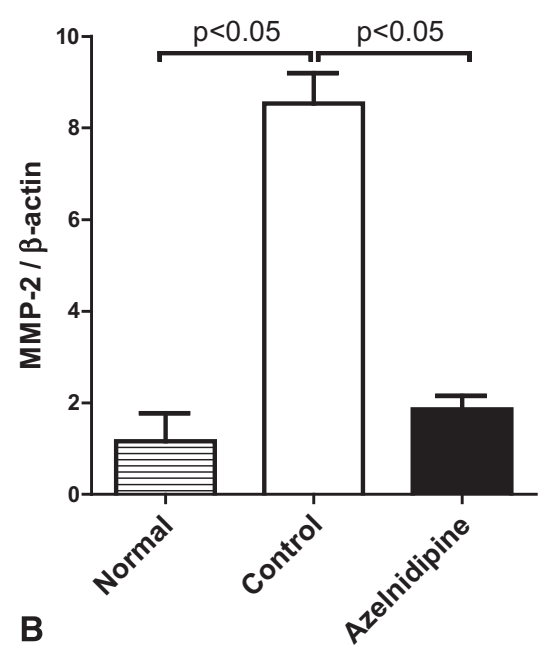

SIRT-1

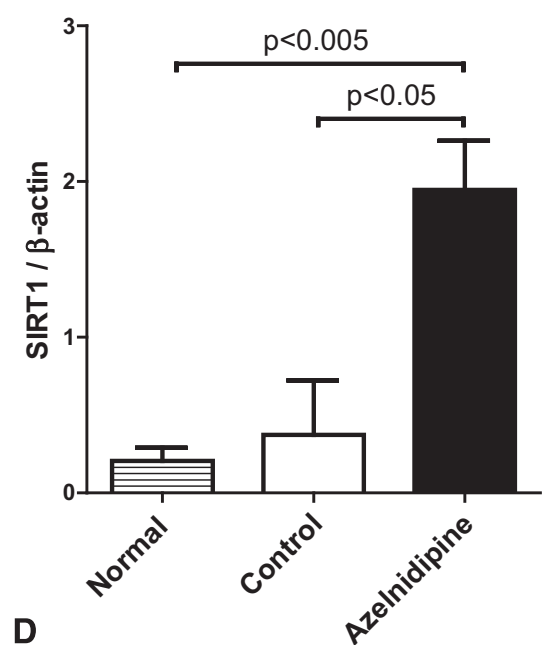

FIGURE 5. Gene expression levels of TNF- $\alpha$, MMP-2, MMP-9, and SIRT- 1 in aortic wall. Real-time PCR analyses of (A) TNF- $\alpha$, (B) MMP-2, (C) MMP-9, and (D) SIRT-1 were performed in mice treated with combination infusion of angiotensin II and BAPN only (control group), mice administered azelnidipine and combination infusion (azelnidipine group), and mice treated with placebo vehicle (normal group). Values are expressed as mean $\pm \mathrm{standard} \mathrm{error} \mathrm{(} \mathrm{n}=7 \mathrm{in}$ each group). $T N F-\alpha$, Tumor necrosis factor-alpha; $M M P$, matrix metalloproteinase; $R T-P C R$, reverse-transcriptase polymerase chain reaction; $B A P N$, $\beta$-aminopropionitrile.

the completion experiments showed a higher survival of $91 \%(12 / 13)$. Additionally, $11(91 \%)$ of the 12 mice that survived had AAs at 4 weeks. One of the aims of this study was to establish a unique animal model of degenerative AA in C57BL/6J mice without genetic modification with high reproducibility and low mortality rate. Herein, we have developed a model that achieves those goals and enables future in vivo study of the pathogenesis of aortic aneurysmal disease and evaluation of pharmacologic interventions.

The pathophysiology and molecular pathway by which AAs develop, progress, and eventually rupture in humans remain unclear. The aortic wall consists of 3 layers: the intima, media, and adventitia. The extracellular matrix is composed primarily of elastin and collagen, both of which are important components of the media and adventitia. Elastin is found predominantly in the medial layer, whereas collagen is concentrated in the adventitia. It has previously been demonstrated that elastin degradation in the adventitia may be the most important factor in the rupture of AAs. ${ }^{29,30}$ Degeneration of aortic wall layers by inflammatory stimuli and/or aging results in the progression of aortic aneurysmal disease in humans. ${ }^{29,30}$ Although a previous study showed a correlation between 
the degeneration of arterial wall and the progression of $\mathrm{AA},{ }^{16}$ the results presented here reveal a similar pathophysiology in degenerative AA formation.

Calcium antagonists are often used clinically for hypertensive patients with AAs. Azelnidipine, a calcium antagonist, was previously reported to have some antiinflammatory effect in vitro, ${ }^{19,20,22}$ but there are few studies that demonstrate its effects on AA progression in vivo. In the present study, azelnidipine drastically inhibited the progression of drug-induced mouse degenerative AA independent of its antihypertensive effect. We found that azelnidipine administration significantly inhibited macrophage infiltration into the aortic wall and the periaortic adipose tissue and also decreased the gene expression levels of inflammatory cytokines within the aortic wall. Macrophages produce inflammatory cytokines such as monocyte chemotactic protein 1, interleukin 6 , TNF- $\alpha$, and MMPs, ${ }^{31,32}$ and in perivascular adipose tissues, they have been shown to be strongly associated with atherosclerosis progression. Inasmuch as AA disease is considered in part a chronic inflammatory disease, it is clear that macrophages play a crucial role in the progression of aortic remodeling in the presence of aneurysmal dilatation. ${ }^{33,34}$ Our results suggest that azelnidipine has the anti-inflammatory effect that alters aortic remodeling, which is consistent with previous studies demonstrating the anti-inflammatory effect of azelnidipine in vitro. ${ }^{19-22}$

Although azelnidipine had the anti-inflammatory effect in our model as evidenced by the decreased expression levels of TNF- $\alpha$, MMP-2, and MMP-9, it conversely enhanced gene expression level of vascular SIRT-1. Vascular SIRT-1 possesses cardiovascular protective properties ${ }^{26}$ and improves the function of vascular smooth muscle and vascular endothelial cells. ${ }^{35,36}$ Schug and associates ${ }^{37}$ demonstrated that myeloid deletion of SIRT-1 induces inflammatory signaling in vivo, and SIRT-1 exerts biological actions as an anti-inflammatory molecule. Our data suggest that the anti-inflammatory actions of azelnidipine may be partly mediated by regulation of SIRT- 1 expression in the aortic wall. Importantly, the role of SIRT- 1 needs further clarification in this model, inasmuch as its mechanism and its role independent of BAPN/angiotensin administration in mice treated with azelnidipine alone are currently unknown.

In conclusion, the combination of hypertension and BAPN-induced degeneration of the elastic lamina can lead to degenerative AAs in a mouse model with high reproducibility and low mortality rate. Azelnidipine suppresses the formation of aortic aneurysmal disease via its antiinflammatory effect within the aortic wall and periaortic tissue. These findings suggest that azelnidipine may be useful as a novel pharmacologic agent to prevent the progression of AAs.
We are grateful to Mayuko Higashida MS (The University of Tokushima), Yasushi Yoshida, PhD (The University of Tokushima), Taisuke Nakayama MD (The University of Tokushima), Kenichi Aihara MD, PhD (The University of Tokushima), Michio Shimabukuro MD, PhD (The University of Tokushima), and Toshio Matsumoto MD, PhD (The University of Tokushima).

\section{References}

1. Sakata R, Fujii Y, Kuwano H. Thoracic and cardiovascular surgery in Japan during 2008: annual report by The Japanese Association for Thoracic Surgery. Gen Thorac Cardiovasc Surg. 2010;58:356-83.

2. Lewiss RE, Egan DJ, Shreves A. Vascular abdominal emergencies. Emerg Med Clin North Am. 2011;29:253-72. viii.

3. Assar AN, Zarins CK. Ruptured abdominal aortic aneurysm: a surgical emergency with many clinical presentations. Postgrad Med J. 2009;85:268-73.

4. Prinssen M, Verhoeven EL, Buth J, Cuypers PW, van Sambeek MR, Balm R, et al. Dutch Randomized Endovascular Aneurysm Managemen (DREAM)Trial Group. A randomized trial comparing conventional and endovascular repair of abdominal aortic aneurysms. N Engl J Med. 2004;351: 1607-18.

5. De Bruin JL, Baas AF, Buth J, Prinssen M, Verhoeven EL, Cuypers PW, et al Long-term outcome of open or endovascular repair of abdominal aortic aneurysm. $N$ Engl J Med. 2010;362:1881-9.

6. Braverman AC. Medical management of thoracic aortic aneurysm disease. J Thorac Cardiovasc Surg. 2013;145:S2-6.

7. Isselbacher EM. Thoracic and abdominal aortic aneurysms. Circulation. 2005; 111:816-28

8. Krishna SM, Dear AE, Norman PE, Golledge J. Genetic and epigenetic mechanisms and their possible role in abdominal aortic aneurysm. Atherosclerosis. 2010;212:16-29

9. Tang PC, Coady MA, Lovoulos C, Dardik A, Aslan M, Elefteriades JA, et al. Hyperplastic cellular remodeling of the media in ascending thoracic aortic aneurysms. Circulation. 2005;112:1098-105.

10. Sawabe M. Vascular aging: from molecular mechanism to clinical significance. Geriatr Gerontol Int. 2010;10(Suppl 1):S213-20.

11. Lopez-Candales A, Holmes DR, Liao S, Scott MJ, Wickline SA, Thompson RW. Decreased vascular smooth muscle cell density in medial degeneration of human abdominal aortic aneurysms. Am J Pathol. 1997;150:993-1007.

12. Schlatmann TJ, Becker AE. Histologic changes in the normal aging aorta: implications for dissecting aortic aneurysm. Am J Cardiol. 1977;39:13-20.

13. Sibon I, Sommer P, Lamaziere JM, Bonnet J. Lysyl oxidase deficiency: a new cause of human arterial dissection. Heart. 2005;91:e33.

14. Behmoaras J, Slove S, Seve S, Vranckx R, Sommer P, Jacob MP. Differential expression of lysyl oxidases LOXL1 and LOX during growth and aging suggests specific roles in elastin and collagen fiber remodeling in rat aorta. Rejuvenation Res. 2008;11:883-9.

15. Yanagisawa H, Davis EC. Unraveling the mechanism of elastic fiber assembly: the roles of short fibulins. Int J Biochem Cell Biol. 2010;42:1084-93.

16. Kanematsu Y, Kanematsu M, Kurihara C, Tsou TL, Nuki Y, Liang EI, et al Pharmacologically induced thoracic and abdominal aortic aneurysms in mice. Hypertension. 2010;55:1267-74.

17. McCallum HM. Experimental lathyrism in mice. J Pathol Bacteriol. 1965;89: 625-36.

18. McCallum HM. Experimental lathyrism in tissue culture. J Pathol Bacteriol. 1965;89:637-44

19. Matsui T, Yamagishi S, Nakamura K, Inoue H. Azelnidipine, a new long-acting calcium-channel blocker, inhibits tumour necrosis factor-alpha-induced monocyte chemoattractant protein-1 expression in endothelial cells. J Int Med Res. 2006;34:671-5.

20. Naito Y, Shimozawa M, Manabe H, Nakabe N, Katada K, Kokura S, et al. Azelnidipine, a new calcium channel blocker, inhibits endothelial inflammatory response by reducing intracellular levels of reactive oxygen species. Eur J Pharmacol. 2006;546:11-8.

21. Nakano K, Egashira K, Ohtani K, Gang Z, Iwata E, Miyagawa M, et al. Azelnidipine has anti-atherosclerotic effects independent of its blood pressure-lowering actions in monkeys and mice. Atherosclerosis. 2008;196:172-9.

22. Shinomiya K, Mizushige K, Fukunaga M, Masugata H, Ohmori K, Kohno M, et al. Antioxidant effect of a new calcium antagonist, azelnidipine, in cultured human arterial endothelial cells. J Int Med Res. 2004;32:170-5. 
23. Weiss D, Kools JJ, Taylor WR. Angiotensin II-induced hypertension accelerates the development of atherosclerosis in apoE-deficient mice. Circulation. 2001; 103:448-54.

24. Gletsu N, Doan TN, Cole J, Sutliff RL, Bernstein KE. Angiotensin II-induced hypertension in mice caused an increase in insulin secretion. Vascul Pharmacol. 2005;42:83-92.

25. Jinno T, Iwai M, Li Z, Li JM, Liu HW, Cui TX, et al. Calcium channel blocker azelnidipine enhances vascular protective effects of AT1 receptor blocker olmesartan. Hypertension. 2004;43:263-9.

26. Guarente L. Franklin H. Epstein Lecture: Sirtuins, aging, and medicine. N Engl J Med. 2011;364:2235-44.

27. Collins CG, Leahy AL. Screening of abdominal aortic aneurysms. Surgeon. 2006;4:83-5.

28. Cronenwett JL. Screening for abdominal aortic aneurysms. Ann Intern Med. 2005;143:309. author reply 309-10.

29. Sakalihasan N, Limet R, Defawe OD. Abdominal aortic aneurysm. Lancet. 2005; $365: 1577-89$.

30. Weintraub NL. Understanding abdominal aortic aneurysm. N Engl J Med. 2009; 361:1114-6.
31. Ley K, Miller YI, Hedrick CC. Monocyte and macrophage dynamics during atherogenesis. Arterioscler Thromb Vasc Biol. 2011;31:1506-16.

32. Hansson GK, Hermansson A. The immune system in atherosclerosis. Nature Immunol. 2011;12:204-12.

33. Nuki Y, Matsumoto MM, Tsang E, Young WL, van Rooijen N, Kurihara C, et al. Roles of macrophages in flow-induced outward vascular remodeling. J Cereb Blood Flow Metab. 2009;29:495-503.

34. Ota R, Kurihara C, Tsou TL, Young WL, Yeghiazarians Y, Chang M, et al. Roles of matrix metalloproteinases in flow-induced outward vascular remodeling. J Cereb Blood Flow Metab. 2009;29:1547-58.

35. Donato AJ, Magerko KA, Lawson BR, Durrant JR, Lesniewski LA, Seals DR. SIRT-1 and vascular endothelial dysfunction with ageing in mice and humans. J Physiol. 2011;589(Pt 18):4545-54.

36. Takemura A, Iijima K, Ota H, Son BK, Ito Y, Ogawa S, et al. Sirtuin 1 retards hyperphosphatemia-induced calcification of vascular smooth muscle cells. Arterioscler Thromb Vasc Biol. 2011;31:2054-62.

37. Schug TT, Xu Q, Gao H, Peres-da-Silva A, Draper DW, Fessler MB, et al. Myeloid deletion of SIRT1 induces inflammatory signaling in response to environmental stress. Mol Cell Biol. 2010;30:4712-21. 